\title{
Patch near field acoustic holography based on particle velocity measurements
}

\author{
Zhang, Yong-Bin; Jacobsen, Finn; Bi, Chuan-Xing; Chen, Xin-Zhao
}

Published in:

Acoustical Society of America. Journal

Link to article, DOI:

$10.1121 / 1.3158819$

Publication date:

2009

Document Version

Publisher's PDF, also known as Version of record

Link back to DTU Orbit

Citation (APA):

Zhang, Y-B., Jacobsen, F., Bi, C-X., \& Chen, X-Z. (2009). Patch near field acoustic holography based on particle velocity measurements. Acoustical Society of America. Journal, 126(2), 721-727.

https://doi.org/10.1121/1.3158819

\section{General rights}

Copyright and moral rights for the publications made accessible in the public portal are retained by the authors and/or other copyright owners and it is a condition of accessing publications that users recognise and abide by the legal requirements associated with these rights.

- Users may download and print one copy of any publication from the public portal for the purpose of private study or research.

- You may not further distribute the material or use it for any profit-making activity or commercial gain

- You may freely distribute the URL identifying the publication in the public portal

If you believe that this document breaches copyright please contact us providing details, and we will remove access to the work immediately and investigate your claim. 


\title{
Patch near field acoustic holography based on particle velocity measurements
}

\author{
Yong-Bin Zhang ${ }^{\text {a) }}$ \\ Institute of Sound and Vibration Research, Hefei University of Technology, Hefei 230009, China \\ Finn Jacobsen \\ Department of Electrical Engineering, Acoustic Technology, Technical University of Denmark, Building 352, \\ $\emptyset$ rsteds Plads, DK-2800 Kongens Lyngby, Denmark \\ Chuan-Xing Bi and Xin-Zhao Chen \\ Institute of Sound and Vibration Research, Hefei University of Technology, Hefei 230009, China
}

(Received 3 April 2009; revised 21 April 2009; accepted 15 May 2009)

\begin{abstract}
Patch near field acoustic holography (PNAH) based on sound pressure measurements makes it possible to reconstruct the source field near a source by measuring the sound pressure at positions on a surface that is comparable in size to the source region of concern. Particle velocity is an alternative input quantity for $\mathrm{NAH}$, and the advantage of using the normal component of the particle velocity rather than the sound pressure as the input of conventional spatial Fourier transform based NAH and as the input of the statistically optimized variant of NAH has recently been demonstrated. This paper examines the use of particle velocity as the input of PNAH. Because the particle velocity decays faster toward the edges of the measurement aperture than the pressure does and because the wave number ratio that enters into the inverse propagator from pressure to velocity amplifies high spatial frequencies, PNAH based on particle velocity measurements can give better results than the pressure-based PNAH with a reduced number of iterations. A simulation study, as well as an experiment carried out with a pressure-velocity sound intensity probe, demonstrates these findings. (C) 2009 Acoustical Society of America. [DOI: 10.1121/1.3158819]
\end{abstract}

PACS number(s): 43.60.Pt, 43.20.Ye, 43.40.At [EGW]

Pages: $721-727$

\section{INTRODUCTION}

Near field acoustic holography (NAH) is a useful technique for visualizing sound fields near complicated sources of sound. In addition to the classical NAH technique based on spatial discrete Fourier transforms, ${ }^{1-3}$ many alternative methods have been developed in the past years, e.g., the inverse boundary element method, ${ }^{4-6}$ the statistically optimized method, ${ }^{7,8}$ the Helmholtz equation least-squares method, ${ }^{9,10}$ and the equivalent source method. ${ }^{11-13}$ Among all these techniques NAH based on spatial Fourier transforms is the easiest to implement and also the most computationally efficient because of the use of the fast Fourier transform algorithm. However, the spatial Fourier transform method requires that the measurement aperture covers a large region where the level drops to a sufficiently low value near the edges in order to reduce truncation effects. Often it is not possible to measure the sound field over such a large aperture, and even when it is possible it is likely to be very uneconomical. If the measurement aperture is not large enough, the reconstruction results will be significantly affected by the truncation effect; wrap-around errors and other errors due to the discontinuity of the aperture edge will be introduced.

In order to overcome this problem a technique called patch NAH (PNAH) has been proposed. In PNAH the sound

\footnotetext{
${ }^{\text {a) }}$ Author to whom correspondence should be addressed. Electronic mail: zybmy1997@163.com
}

field is only measured on a surface that is approximately as large as the patch on the source surface where the reconstruction is required. The key problem of PNAH is a numerical tangential extension of the measurement aperture. Saijyou and Yoshikawa ${ }^{14}$ proposed a patch approach based on an iterative procedure, Williams and co-workers ${ }^{15-17}$ extended it by using singular value decomposition and improved its accuracy by regularization, Sarkissian ${ }^{18,19}$ developed a PNAH method using the superposition method, and Lee and Bolton $^{20}$ investigated PNAH in a cylindrical geometry. Yet another PNAH technique has been proposed by Steiner and Hald. $^{7}$

All these techniques are based on measurement of the sound pressure. However, since a particle velocity transducer is available, ${ }^{21}$ PNAH based on measurement of the normal component of the particle velocity is investigated in this paper. The use of the particle velocity in spatial Fourier transform based NAH has been examined by Jacobsen and Liu and found to be advantageous. ${ }^{22}$ Statistically optimized NAH based on measurement of particle velocity has also been investigated, and the results showed a similar advantage. ${ }^{23}$

Compared with PNAH based on measurement of the sound pressure one might expect two advantages in using the normal component of the particle velocity. The normal component of the particle velocity generated by vibrations in a local source area decays faster toward the edges of the measurement aperture than the sound pressure does, and the particle velocity can therefore in general be expected to be "more local" than the pressure; thus the extrapolation of the 


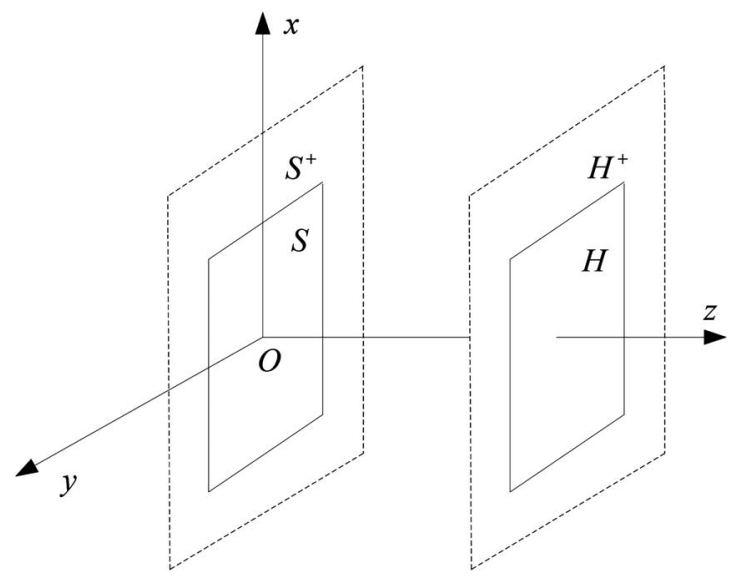

FIG. 1. Source and hologram plane.

sound field in measurement aperture might be expected to be more successful. Another potential advantage is associated with inherent properties of the inverse pressure-to-velocity propagator described in Ref. 22. The purpose of this paper is to examine the matter.

\section{PNAH BASED ON MEASUREMENT OF PARTICLE VELOCITY}

The derivation of the theory of PNAH based on measurement of the particle velocity follows the derivation for pressure-based PNAH in Ref. 15. Let the vector $\mathbf{v}_{h}$ represent the measured particle velocities at points $\mathbf{r}_{h}$ in a patch $H$, which is smaller than the sound source (see Fig. 1). The purpose is now to reconstruct the pressure and the normal component of the velocity at points $\mathbf{r}_{s}$ in a region $S$ with the same size as $H$ as accurately as possible. If the reconstruction is made directly using a spatial Fourier transform the result will be contaminated seriously by errors caused by the finite measurement aperture. These errors will be reduced if the measured particle velocity can be extrapolated into the region $\mathrm{H}^{+}$. An iterative procedure for such a continuation of the measurement is described in Ref. 15. First the measured particle velocity is zero-padded,

$$
\mathbf{v}_{h}^{(0)}=\left\{\begin{array}{cc}
\mathbf{v}_{h} & \left(\mathbf{r}_{h} \in H\right) \\
0 & \left(\mathbf{r}_{h} \in H^{+}\right),
\end{array}\right.
$$

where $\mathbf{v}_{h}^{0}$ is the initial value of the iteration. The relationship between the filtered particle velocity $\widetilde{\mathbf{v}}_{h}$ and the corresponding measured particle velocity for the $i$ th iteration can be written as

$$
\widetilde{\mathbf{v}}_{h}^{(i)}=\mathbf{F}^{-1}\left\{F_{\alpha}^{(i)} \mathbf{F}\left\{\mathbf{v}_{h}^{(i)}\right\}\right\},
$$

where $\mathbf{F}$ and $\mathbf{F}^{-1}$ represent the discrete two-dimensional spatial Fourier transform and its inverse, and the filter factor $F_{\alpha}^{(i)}$ for the $i$ th iteration is

$$
F_{\alpha}^{(i)}=\frac{\left|G\left(k_{x}, k_{y}\right)\right|^{2}}{\left|G\left(k_{x}, k_{y}\right)\right|^{2}+\alpha^{(i)}\left(\frac{\alpha^{(i)}}{\alpha^{(i)}+\left|G\left(k_{x}, k_{y}\right)\right|^{2}}\right)^{2}} .
$$

In this equation $G\left(k_{x}, k_{y}\right)=e^{-j k_{z} d}$ is the propagator between the velocity of the surface of the source and the particle velocity in the hologram plane, $d$ is the distance between the hologram plane and reconstruction plane (with the $e^{j \omega t}$ sign convention), the wave number component in the $z$-direction is given by

$$
k_{z}=\left\{\begin{array}{ccc}
\sqrt{k^{2}-k_{x}^{2}-k_{y}^{2}} & \text { for } & k_{x}^{2}+k_{y}^{2} \leq k^{2} \\
-j \sqrt{k_{x}^{2}+k_{y}^{2}-k^{2}} & \text { for } & k_{x}^{2}+k_{y}^{2}>k^{2},
\end{array}\right.
$$

and $\alpha^{(i)}$ is the regularization parameter of the $i$ th iteration, determined by the Morozov discrepancy principle ${ }^{24}$ using

$$
\left(\widetilde{\mathbf{v}}_{h}^{(i)}-\mathbf{v}_{h}^{(i)}\right)^{H}\left(\widetilde{\mathbf{v}}_{h}^{(i)}-\mathbf{v}_{h}^{(i)}\right) / M=\left(\sigma^{(i)}\right)^{2},
$$

where $M$ is the total number of points in the region $\mathrm{H}+\mathrm{H}^{+}$, and $\sigma^{(i)}$ is the standard deviation of the noise of the $i$ th iteration, which is estimated as described in Ref. 24. In each iteration the regularization parameter can be determined by varying $\alpha^{(i)}$ until Eq. (5) is satisfied within a given tolerance. Here an alternative, fast method is used to determine $\alpha^{(i)}$ by searching for the minimum between the left part and the right part of Eq. (5) (using the function "fminbnd" of MATLAB).

The iteration runs as follows. Equations (1) and (2) give the filtered initial particle velocity. The input particle velocity for the first iteration is obtained by replacing the particle velocity in the region $H$ with the original measured particle velocity $\mathbf{v}_{h}$, etc., that is,

$$
\mathbf{v}_{h}^{(i+1)}=\left\{\begin{array}{cc}
\mathbf{v}_{h} & \left(\mathbf{r}_{h} \in H\right) \\
\widetilde{\mathbf{v}}_{h}^{(i)} & \left(\mathbf{r}_{h} \in H^{+}\right) .
\end{array}\right.
$$

The iteration can be stopped if the following condition is satisfied:

$$
\frac{\left\|\widetilde{\mathbf{v}}_{h}^{(i+1)}-\widetilde{\mathbf{v}}_{h}^{(i)}\right\|_{2}}{\left\|\widetilde{\mathbf{v}}_{h}^{(i)}\right\|_{2}}<\varepsilon,
$$

where $\|\bullet\|_{2}$ represents the $L 2$-norm of a vector and $\varepsilon>0$ is a small number (for example, $\varepsilon=10^{-5}$ ). Finally, the velocity of the source, $\mathbf{v}_{s}$, and the pressure on the surface of the source, $\mathbf{p}_{s}$, can be reconstructed from the filtered particle velocity and its continuation into the region $\mathrm{H}^{+}$after the iteration process has converged,

$$
\begin{aligned}
& \mathbf{v}_{s}=\mathbf{F}^{-1}\left\{\mathbf{G}^{-1} F_{\alpha}^{(i)} \mathbf{F}\left\{\widetilde{\mathbf{v}}_{h}^{(i)}\right\}\right\}, \\
& \mathbf{p}_{s}=\mathbf{F}^{-1}\left\{\mathbf{G}_{N}^{-1} F_{\alpha}^{(i)} \mathbf{F}\left\{\widetilde{\mathbf{v}}_{h}^{(i)}\right\}\right\},
\end{aligned}
$$

where $\mathbf{G}^{-1}\left(k_{x}, k_{y}\right)=e^{i k_{z} d}$ is the inverse velocity propagator, and

$$
G_{N}^{-1}=\rho c\left(k / k_{z}\right) e^{j k_{z} d}
$$

is the inverse of the propagator between the pressure in the source plane and the particle velocity in the hologram plane and $\rho c$ is the characteristic impedance of the medium. Note that the velocity-to-pressure propagator $\mathbf{G}_{N}^{-1}$ has a singularity near the radiation circle where $k_{z}$ tends to zero. To reduce the singularity the propagator $\mathbf{G}_{N}^{-1}$ should be smoothed as described in Ref. 25.

Y. Zhang and F. Jacobsen: Patch near field acoustic holography 
(a)

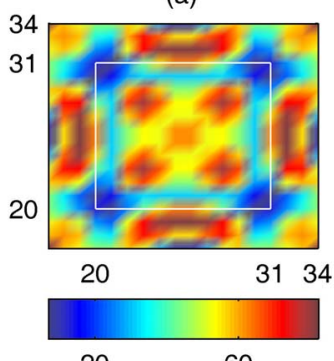

20

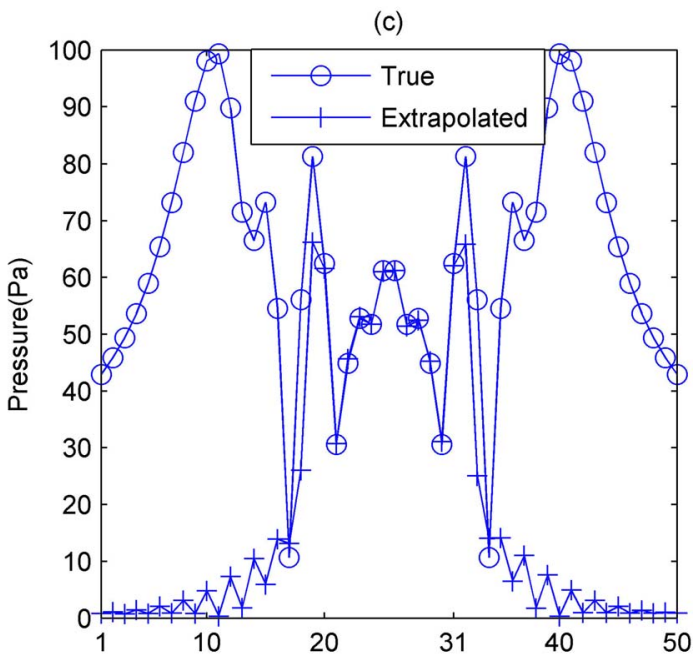

FIG. 2. (Color online) Extrapolation of the sound pressure at $2 \mathrm{kHz}$ : (a) true pressure, (b) extrapolated pressure, and (c) comparison along a line. The frame in (a) and (b) indicates the boundary of the patch.

\section{A SIMULATION STUDY}

To examine the performance of the method described in the foregoing and compare it with the original version based on extrapolation of the sound pressure a simulation study has been carried out. The test case was a point driven simply supported 3-mm-thick aluminum plate in an infinite baffle. The dimensions of the plate were $0.58 \times 0.58 \mathrm{~m}^{2}$, and the excitation of the plate was a harmonic force with an amplitude of $100 \mathrm{~N}$ acting at the center of the plate. The reconstruction plane was located at $z=0.02 \mathrm{~m}$, and the hologram plane was located at $z=0.05 \mathrm{~m}$. The velocity of the surface of the plate was calculated by a modal sum, and the radiated sound field was calculated from a numerical approximation to Rayleigh's first integral. The sound pressure and the particle velocity were computed in the hologram plane in a grid $12 \times 12$ points with a lattice spacing in the $x$ - and $y$-direction of $0.02 \mathrm{~m}$. Thus, the measured data covered an area of $0.22 \times 0.22 \mathrm{~m}^{2}$, which is much smaller than the plate. The iteration procedure was applied both to the particle velocity and to the sound pressure in this patch in order to extrapolate the data to a region of $50 \times 50$ points with dimensions of $0.98 \times 0.98 \mathrm{~m}^{2}$. To make the simulation study more realistic noise has been added to the measured data corresponding to a signal-to-noise ratio of $35 \mathrm{~dB}$. In the simulation the number $\varepsilon$ in the convergence condition is $10^{-3}$. (a)
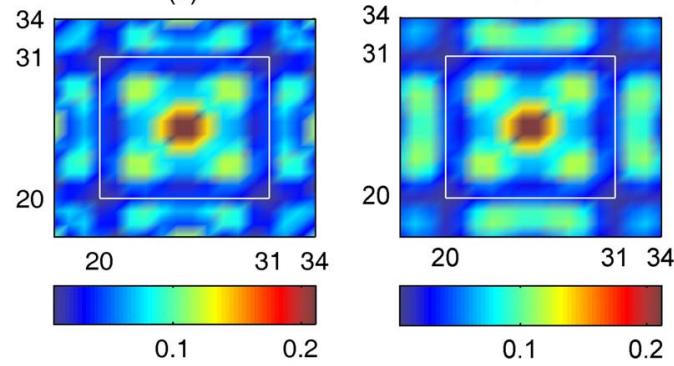

(c)

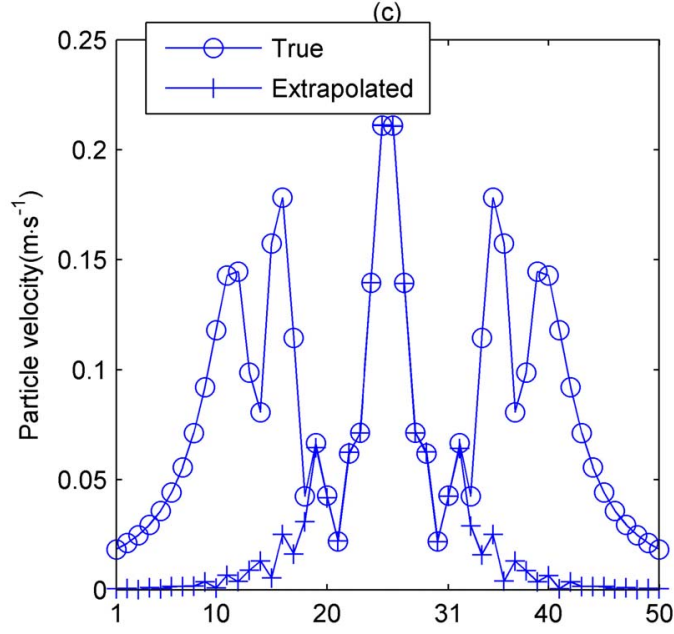

FIG. 3. (Color online) Extrapolation of the particle velocity at $2 \mathrm{kHz}$ : (a) true velocity, (b) extrapolated velocity, and (c) comparison along a line. The frame in (a) and (b) indicates the boundary of the patch.

\section{A. Extrapolation of sound field}

Figures 2 and 3 compare the true and extrapolated sound pressure and particle velocity in the hologram plane at 2 $\mathrm{kHz}$. Within the patch both the extrapolated sound pressure and particle velocity agree well with the true values. However, in the adjacent region outside the patch, the extrapolated pressure is in very poor agreement with the true pressure. The extrapolated particle velocity is more accurate than the extrapolated pressure, and, as can be seen in Fig. 3(b), reasonable agreement is obtained at the first two points outside the patch. However, it is clear that the region where the particle velocity can be extended accurately is limited. One may define the relative errors as

$$
\begin{aligned}
& E_{p}=\frac{\left\|p_{\text {true }}-p_{\text {extrapolated }}\right\|_{2}}{\left\|p_{\text {true }}\right\|_{2}} \times 100 \%, \\
& E_{v}=\frac{\left\|v_{h, \text { true }}-v_{h, \text { extrapolated }}\right\|_{2}}{\left\|v_{h, \text { true }}\right\|_{2}} \times 100 \% .
\end{aligned}
$$

In a surrounding region one point, two points, and three points outside the patch the errors of the extrapolated sound pressure are $24.7 \%, 65.8 \%$, and $91.3 \%$, and the corresponding errors of the particle velocity are $17.4 \%, 32.8 \%$, and $75.7 \%$.

In addition to the increased reliability the extrapolation based on the particle velocity has another advantage, the iteration speed; for the same convergence condition, the re- 
(a)

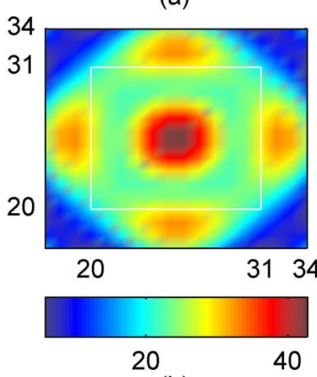

(b)

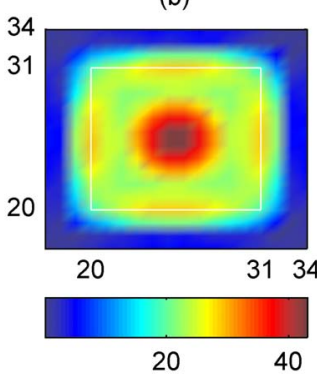

(c)

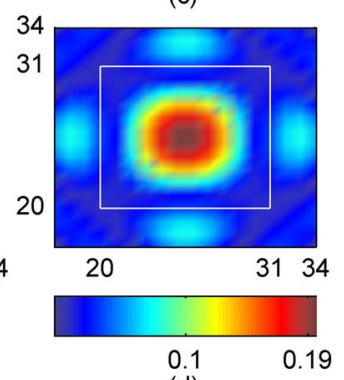

(d)

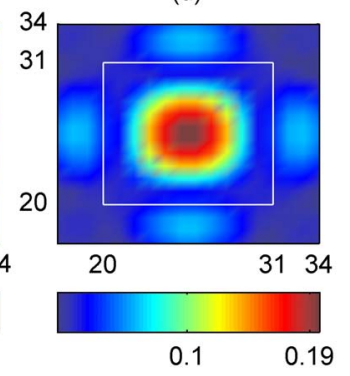

FIG. 4. (Color online) Extrapolation of sound pressure and particle velocity at $800 \mathrm{~Hz}$ : (a) true pressure, (b) extrapolated pressure, (c) true particle velocity, and (d) extrapolated particle velocity.

quired number of iterations for extrapolating the sound pressure was 415 in the example described above, whereas it was 203 for extrapolating the particle velocity.

It can be concluded that the particle velocity can lead to better extrapolation results than the pressure with less iterations. However, the number of required iterations for extrapolating the particle velocity is not always much smaller than the required number for extrapolating the pressure; for example, for the same test case at $800 \mathrm{~Hz}$, the required numbers of iterations are 237 for the particle velocity and 271 for the pressure. Figure 4 compares the true and extrapolated sound pressure and particle velocity at $800 \mathrm{~Hz}$. It can be seen that the extrapolated particle velocity is in better agreement with the true values than the extrapolated pressure is, although the particle velocity and the pressure require a similar number of iterations to converge. The errors defined as above are $31.8 \%, 85.1 \%$, and $78.2 \%$ for the pressure and $14.1 \%$, $26.0 \%$, and $42.9 \%$ for the particle velocity. Other cases (not shown) have confirmed the tendency.

A better extrapolation of the pressure can be obtained if the number of the iterations is increased significantly. The extrapolated pressures at 2000 and $800 \mathrm{~Hz}$ after 1000 itera-

(a)

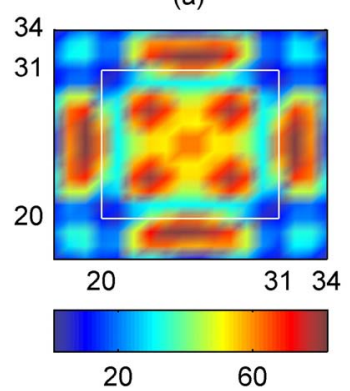

(b)

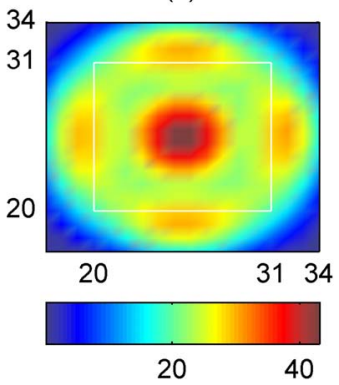

FIG. 5. (Color online) Extrapolation of the sound pressure at $2 \mathrm{kHz}$ (a) and $800 \mathrm{~Hz}$ (b) after 1000 iterations.
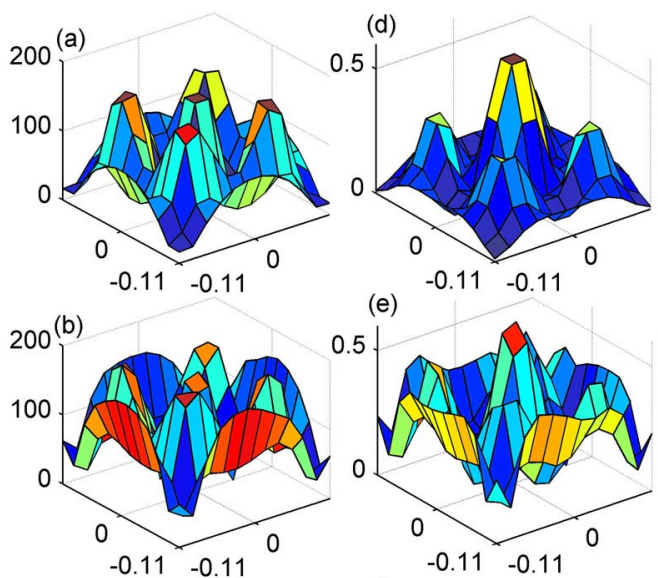

(e)
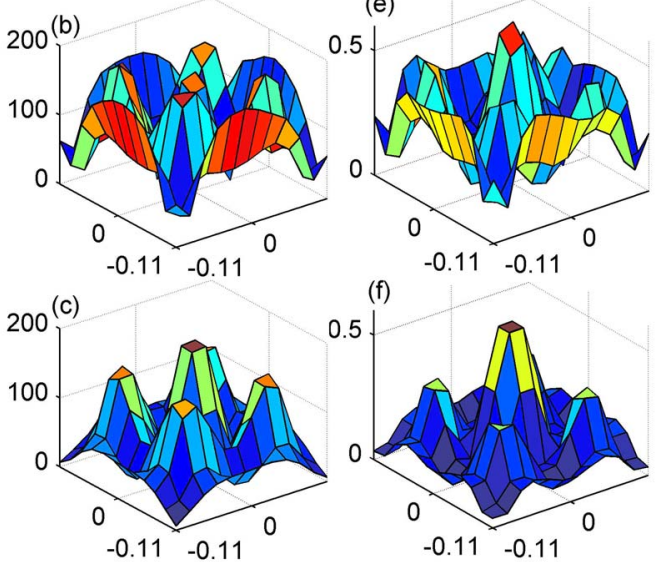

FIG. 6. (Color online) Reconstruction at $2 \mathrm{kHz}$ : (a) true pressure, (b) pressure-to-pressure, (c) velocity-to-pressure, (d) true velocity, (e) pressureto-velocity, and (f) velocity-to-velocity.

tions are shown in Fig. 5. It can be seen that the accuracy of the extrapolation has been improved, while the computational cost and time have been increased.

\section{B. Reconstructed sound field}

Figure 6 shows the reconstructed pressure and velocity in the source plane at $2 \mathrm{kHz}$. Parts (a), (b), and (c) compare
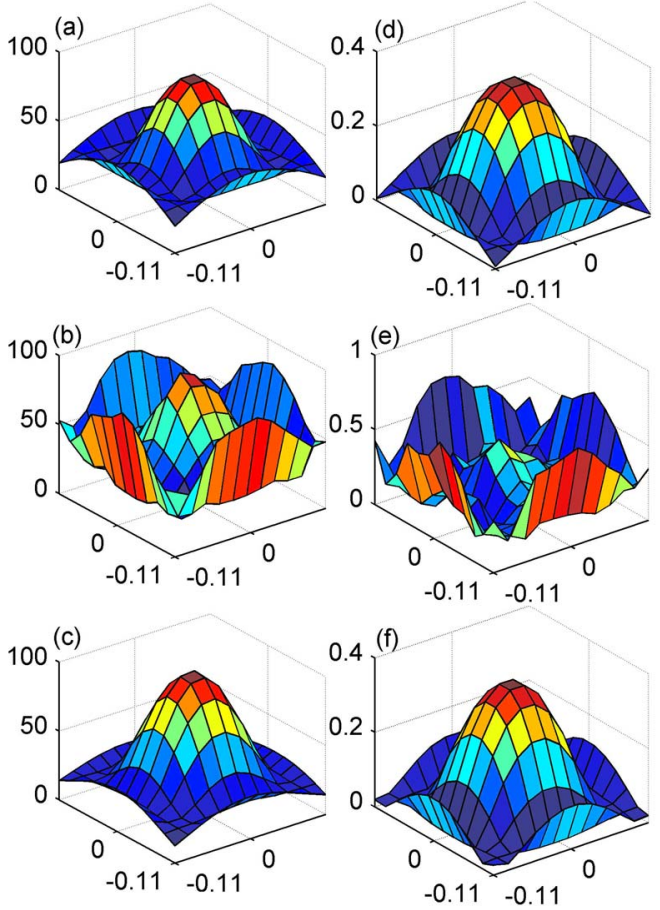

FIG. 7. (Color online) Reconstruction at $800 \mathrm{~Hz}$ : (a) true pressure, (b) pressure-to-pressure, (c) velocity-to-pressure, (d) true velocity, (e) pressureto-velocity, and (f) velocity-to-velocity. 


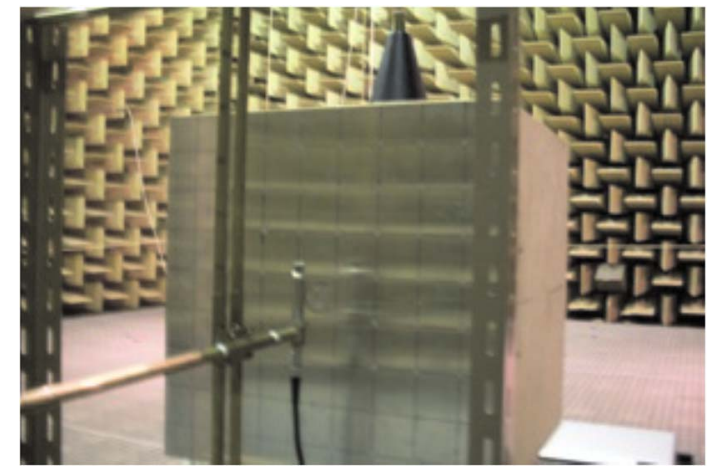

FIG. 8. (Color online) Experimental setup.

the true pressure, the pressure predicted from the extrapolated pressure, and the pressure predicted from the extrapolated particle velocity. It can be seen that the pressure reconstructed from the extrapolated particle velocity is in better agreement with the true pressure than the pressure reconstructed from pressure data, in particular, near the edges of the aperture. Parts (d), (e), and (f) compare the true particle velocity, the particle velocity predicted from the extrapolated pressure, and the particle velocity predicted from the extrapolated particle velocity. It is apparent that the particle velocity reconstructed from the pressure is not very accurate, whereas the reconstruction based on the particle velocity is far better.

Figure 7 shows a similar comparison when the plate is driven at $800 \mathrm{~Hz}$. Again reconstructions based on the extrapolated particle velocity are fairly accurate, and, in particular, the velocity-to-velocity reconstruction is far better than the pressure-to-velocity reconstruction. By increasing the number of iterations for the pressure (as in Fig. 5) better reconstructions can be obtained at the expense of increased computer resources, and in some cases (not shown) the pressure-to-pressure reconstruction was found to be better than the velocity-to-pressure reconstruction, but velocity-tovelocity reconstructions were invariably found to be considerably better than pressure-to-velocity reconstructions.

\section{EXPERIMENTAL RESULTS}

An experiment has been carried out in a large anechoic room at the Technical University of Denmark. The source was a 3-mm-thick aluminum plate with dimensions 44 $\times 44 \mathrm{~cm}^{2}$ mounted as one of the surfaces of a box of heavy fiberboard and excited by a loudspeaker inside the box. The sound pressure and the particle velocity were measured at
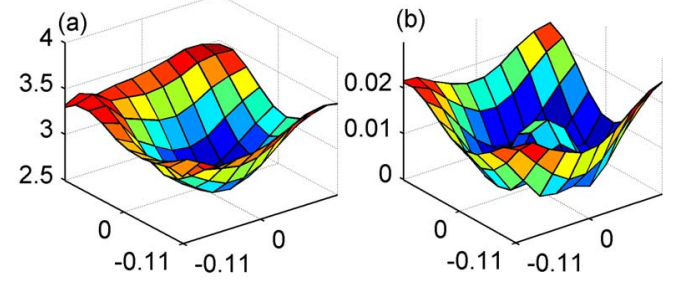

FIG. 9. (Color online) True (a) sound pressure and (b) particle velocity in the reconstruction plane.
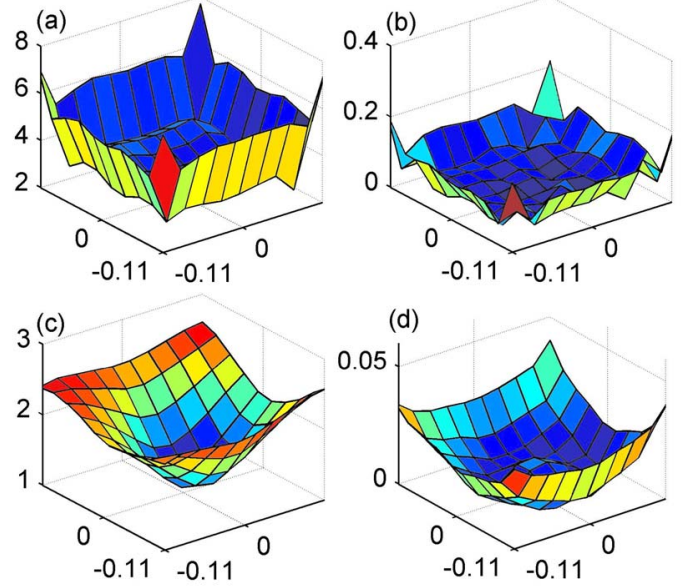

FIG. 10. (Color online) Reconstruction after 1500 iterations: (a) pressureto-pressure, (b) pressure-to-velocity, (c) velocity-to-pressure, and (d) velocity-to-velocity.

$18 \times 19$ points in two planes of dimensions $42.5 \times 45 \mathrm{~cm}^{2}$ using a $\frac{1}{2}$-in. $p$ - $u$ intensity probe produced by Microflown. The plate, the transducer, and the test fixture for the transducer are shown in Fig. 8. The transducer was calibrated as described in Ref. 26. The hologram plane was $8 \mathrm{~cm}$ from the plate, whereas the reconstruction plane was $4.5 \mathrm{~cm}$ from the plate and the measured data in it served as the "true" reference data. A Brüel \& Kjær PULSE analyzer (type 3560) was used for measuring the frequency responses between the pressure and particle velocity signals from the transducer and the signal generated by the PULSE analyzer (pseudorandom noise) for driving the source.

A $10 \times 10$ point small patch with the center coincident with the center of the vibrating plate was selected for the extrapolation and reconstruction. Thus the patch covered an area of $22.5 \times 22.5 \mathrm{~cm}^{2}$. The number $\varepsilon$ in the convergence condition was $10^{-5}$ in this experiment. Figure 9 shows the true pressure and particle velocity in the reconstruction plane at $160 \mathrm{~Hz}$, Figs. 10(a) and 10(b) show the pressure and particle velocity predicted from the pressure in the measurement
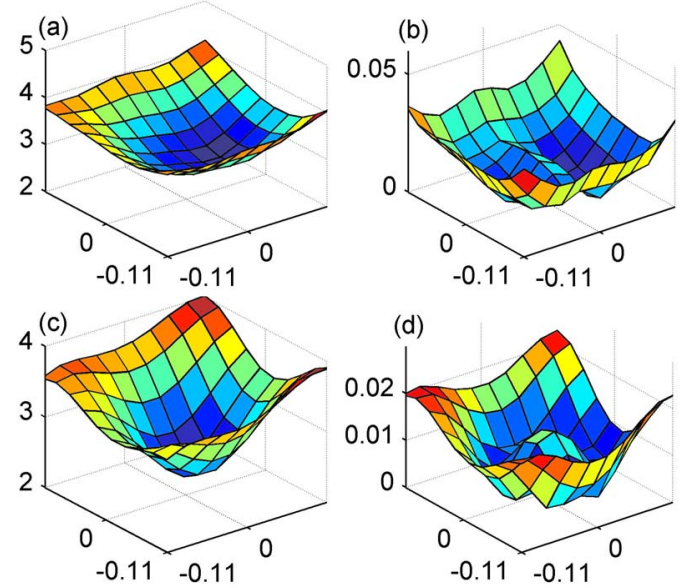

FIG. 11. (Color online) Reconstruction after convergence: (a) pressure-topressure, (b) pressure-to-velocity, (c) velocity-to-pressure, and (d) velocityto-velocity. 
TABLE I. Comparison of reconstructions based on pressure and on particle velocity.

\begin{tabular}{|c|c|c|c|c|c|c|}
\hline \multirow{2}{*}{$\begin{array}{l}\text { Frequency } \\
\qquad(\mathrm{Hz})\end{array}$} & \multicolumn{2}{|c|}{ Percent error of pressure reconstruction } & \multicolumn{2}{|c|}{ Percent error of velocity reconstruction } & \multicolumn{2}{|c|}{ Required number of iterations } \\
\hline & Pressure-to-pressure & Velocity-to-pressure & Pressure-to-velocity & Velocity-to-velocity & Based on pressure & Based on velocity \\
\hline 100 & 10.5 & 8.4 & 54 & 7.1 & 14744 & 4295 \\
\hline 200 & 6.5 & 7.9 & 25.5 & 4.2 & 19282 & 16697 \\
\hline 400 & 7.2 & 24.4 & 43.2 & 13.6 & 26518 & 23027 \\
\hline 800 & 10.0 & 22.3 & 35.1 & 10.4 & 15500 & 13153 \\
\hline 1000 & 15.1 & 12.9 & 60.0 & 17.1 & 29014 & 25932 \\
\hline
\end{tabular}

patch after 1500 iterations, and Figs. 10(c) and 10(d) show the pressure and particle velocity predicted from the particle velocity in the measurement patch after 1500 iterations. It can be seen that the reconstruction based on the extrapolated particle velocity is much better than the reconstruction based on the extrapolated pressure with the same number of iterations.

Figure 11 shows a similar comparison when convergence condition has been reached. It is apparent that the particle velocity performs better than the pressure as the input quantity. The relative error of the reconstructed pressure is $12.3 \%$ when it is based on pressure measurements and $6.13 \%$ when it is based on particle velocity measurements, and the corresponding error of the reconstructed particle velocity is $61.2 \%$ when it is based on pressure measurements and $4.33 \%$ when it is based on particle velocity measurements. It should also be mentioned that the extrapolated particle velocity converged after 20790 iterations, whereas the extrapolated pressure required 28142 iterations to converge. The results at some other frequencies are shown in Table I, which demonstrates that velocity-to-velocity reconstructions are generally better than pressure-to-velocity reconstructions. Sometimes pressure-to-pressure reconstructions are more accurate than velocity-to-pressure reconstructions. In all cases the iterative procedure converges faster for the particle velocity than for the pressure.

\section{CONCLUSIONS}

A method of PNAH based on extrapolating particle velocity data beyond the measurement aperture has been examined and shown to compare favorably with a similar method based on measurement of the sound pressure, both with respect to the number of required iterations and the quality of the resulting reconstructed sound field. These findings have been demonstrated by simulation as well as by an experiment carried out with a pressure-velocity intensity probe.

\section{ACKNOWLEDGMENT}

The authors would like to thank Microflown for lending the $p-u$ sound intensity probe. This work was supported by the National Natural Science Foundation of China (Grant Nos. 50675056 and 10874037) and Fok Ying Tung Education Foundation (Grant No. 111058) and the Program for New Century Excellent Talents in University (Grant No. NCET-08-0767). Additionally, the China Scholarship Council is acknowledged for financial support.
${ }^{1}$ J. D. Maynard, E. G. Williams, and Y. Lee, "Nearfield acoustic holography: I. Theory of generalized holography and development of NAH," J. Acoust. Soc. Am. 78, 1395-1413 (1985).

${ }^{2}$ W. A. Veronesi and J. D. Maynard, "Nearfield acoustic holography (NAH): II. Holographic reconstruction algorithms and computer implementation," J. Acoust. Soc. Am. 81, 1307-1322 (1987).

${ }^{3}$ E. G. Williams, H. D. Dardy, and K. B. Washburn, "Generalized nearfield acoustical holography for cylindrical geometry: Theory and experiment," J. Acoust. Soc. Am. 81, 389-407 (1987).

${ }^{4}$ M. R. Bai, "Application of BEM (boundary element method)-based acoustic holography to radiation analysis of sound sources with arbitrarily shaped geometries," J. Acoust. Soc. Am. 92, 533-549 (1992).

${ }^{5}$ B.-K. Kim and J.-G. Ih, "On the reconstruction of the vibro-acoustic field over the surface enclosing an interior space using the boundary element method," J. Acoust. Soc. Am. 100, 3003-3016 (1996).

${ }^{6}$ S.-C. Kang and J.-G. Ih, "Use of nonsingular boundary integral formulation for reducing errors due to near-field measurements in the boundary element method based near-field acoustic holography," J. Acoust. Soc. Am. 109, 1320-1328 (2001)

${ }^{7}$ R. Steiner and J. Hald, "Near-field acoustical holography without the errors and limitations caused by the use of spatial DFT," Int. J. Acoust. Vib. 6, 83-89 (2001).

${ }^{8}$ Y. T. Cho, J. S. Bolton, and J. Hald, "Source visualization by using statistically optimized nearfield acoustical holography in cylindrical coordinates," J. Acoust. Soc. Am. 118, 2355-2364 (2005).

${ }^{9} \mathrm{Z}$. Wang and S. F. Wu, "Helmholtz equation-least-squares method for reconstructing the acoustic pressure field," J. Acoust. Soc. Am. 102, 20202032 (1997).

${ }^{10} \mathrm{~S}$. F. Wu and J. Yu, "Reconstructing interior acoustic pressure fields via Helmholtz equation least-squares method," J. Acoust. Soc. Am. 104, 2054-2060 (1998).

${ }^{11}$ C.-X. Bi, X.-Z. Chen, and J. Chen, "Sound field separation technique based on equivalent source method and its application in nearfield acoustic holography," J. Acoust. Soc. Am. 123, 1472-1478 (2008).

${ }^{12}$ C.-X. Bi, X.-Z. Chen, and J. Chen, "Nearfield acoustic holography based on the equivalent source method," Sci. China, Ser. E: Technol. Sci. 48, 338-353 (2005).

${ }^{13}$ N. P. Valdivia and E. G. Williams, "Study on the comparison of the methods of equivalent sources and boundary element methods for near-field acoustic holography," J. Acoust. Soc. Am. 120, 3694-3705 (2006).

${ }^{14} \mathrm{~K}$. Saijyou and S. Yoshikawa, "Reduction methods of the reconstruction error for large-scale implementation of near-field acoustical holography," J. Acoust. Soc. Am. 110, 2007-2023 (2001).

${ }^{15}$ E. G. Williams, B. H. Houston, and P. C. Herdic, "Fast Fourier transform and singular value decomposition formulations for patch nearfield acoustical holography," J. Acoust. Soc. Am. 114, 1322-1333 (2003).

${ }^{16} \mathrm{~K}$. Saijyou and H. Uchida, "Data extrapolation method for boundary element method-based near-field acoustical holography," J. Acoust. Soc. Am. 115, 785-796 (2004).

${ }^{17}$ E. G. Williams, "Continuation of acoustic near-fields," J. Acoust. Soc. Am. 113, 1273-1281 (2003).

${ }^{18}$ A. Sarkissian, "Extension of measurement surface in near-field acoustic holography," J. Acoust. Soc. Am. 115, 1593-1596 (2004).

${ }^{19}$ A. Sarkissian, "Method of superposition applied to patch near-field acoustic holography," J. Acoust. Soc. Am. 118, 671-678 (2005).

${ }^{20}$ M. Lee and J. S. Bolton, "Patch near-field acoustical holography in cylindrical geometry," J. Acoust. Soc. Am. 118, 3721-3732 (2005).

${ }^{21}$ F. Jacobsen and H.-E. de Bree, "A comparison of two different sound intensity measurement principles," J. Acoust. Soc. Am. 118, 1510-1517 (2005). 
${ }^{22}$ F. Jacobsen and Y. Liu, "Near field acoustic holography with particle velocity transducers," J. Acoust. Soc. Am. 118, 3139-3144 (2005).

${ }^{23}$ F. Jacobsen and V. Jaud, "Statistically optimized near field acoustic holography using an array of pressure-velocity probe," J. Acoust. Soc. Am. 121, 1550-1558 (2007).

${ }^{24}$ E. G. Williams, "Regularization methods for near-field acoustical holog- raphy," J. Acoust. Soc. Am. 110, 1976-1988 (2001).

${ }^{25}$ E. G. Williams and J. D. Maynard, "Numerical evaluation of the Rayleigh integral for planar radiators using the FFT," J. Acoust. Soc. Am. 72, 20202030 (1982).

${ }^{26} \mathrm{~F}$. Jacobsen and V. Jaud, "A note on the calibration of pressure-velocity sound intensity probes," J. Acoust. Soc. Am. 120, 830-837 (2006). 